\title{
Neuropathologic Findings of Dementia with Lewy Bodies (DLB) in a Population-based Vantaa 85+Study
}

\section{Oinas, Minna}

2009

Oinas , M , Polvikoski , T , Sulkava , R , Myllykangas , L, Juva , K, Notkola , I-L, Rastas , S , Niinisto , L , Kalimo , H \& Paetau , A 2009 , ' Neuropathologic Findings of Dementia with Lewy Bodies (DLB) in a Population-based Vantaa 85+Study ' , Journal of Alzheimer's Disease , vol. 18 , pp. 677-689 . https://doi.org/10.3233/JAD-2009-1169

http://hdl.handle.net/10138/305514

https://doi.org/10.3233/JAD-2009-1169

unspecified

acceptedVersion

Downloaded from Helda, University of Helsinki institutional repository.

This is an electronic reprint of the original article.

This reprint may differ from the original in pagination and typographic detail.

Please cite the original version. 
For the Mini-forum on population- or community based studies of aging and dementia with brain autopsies in the Journal of Alzheimer's Disease

\title{
Neuropathologic findings of Dementia with Lewy bodies (DLB) in a population-based Vantaa $85+$ study
}

\author{
Minna Oinas, $\mathrm{MD}^{1,2}$, Tuomo Polvikoski, $\mathrm{MD}, \mathrm{PhD}^{3}$, Raimo Sulkava, $\mathrm{MD}, \mathrm{PhD}^{4}$, Liisa \\ Myllykangas, $\mathrm{MD}, \mathrm{PhD}^{1,5}$, Kati Juva, $\mathrm{MD}, \mathrm{PhD}^{6}$, Irma-Leena Notkola, $\mathrm{PhD}^{7}$, Sari Rastas, $\mathrm{MD}^{8}$, \\ Leena Niinistö MD, $\mathrm{PhD}^{9}$, Hannu Kalimo, $\mathrm{MD}, \mathrm{PhD}^{1}$, Anders Paetau, $\mathrm{MD}, \mathrm{PhD}^{1}$ \\ Department of ${ }^{1}$ Pathology, ${ }^{2}$ Neurosurgery, and ${ }^{6}$ Psychiatry, University of Helsinki, and Helsinki \\ University Central Hospital, Helsinki, Finland \\ ${ }^{3}$ Institute for Ageing and Health, Newcastle University, Campus for Ageing and Vitality, \\ Newcastle upon Tyne, NE4 5PL, UK \\ ${ }^{4}$ School of Public Health and Clinical Nutrition, University of Kuopio, Kuopio, and Rheumatism \\ Foundation Hospital, Heinola, Finland \\ ${ }^{5}$ Folkhälsan institute of Genetics, Helsinki, Finland \\ ${ }^{7}$ Finnish Information Centre for Register Research, Helsinki, Finland \\ ${ }^{8}$ Kauniala Disabled, War Veterans Hospital, Kauniainen, Finland \\ ${ }^{9}$ Katriina Geriatric Hospital, Vantaa, Finland
}

Running title: Lewy-related pathology in very elderly people

Correspondence and proofs to: Minna Oinas; Department of Pathology, University of Helsinki, P.O.Box 21 (Haartmaninkatu 3), FI-00014 University of Helsinki, Finland; minna.oinas@ helsinki.fi tel; +358 50576 8677, fax; +358 919126551

All authors have contributed to the work, agree with the presented findings, and the work has not been published before nor being considered for publication in another journal.

Procedures involving experiments on human subjects have done in accord with the Helsinki Declaration of 1975.

Disclosure: The authors report no conflicts of interest. 


\section{ABSTRACT}

The consortium on dementia with Lewy bodies (CDLB) has established consensus guidelines for the neuropathologic diagnosis of DLB including the likelihood that the neuropathologic findings associate with the clinical syndrome. Nevertheless, clinico-pathological correlations remain controversial. We applied the consensus guidelines for determining Lewyrelated pathology (LRP) and evaluated the clinical presentation in the prospective, populationbased Vantaa $85+$ study consisting of individuals at least 85 years of age. LRP was seen in $36 \%$ of 304 subjects and categorized as follows: $3 \%$ brainstem-predominant, $14 \%$ limbic, $15 \%$ diffuse neocortical type ( $4 \%$ could not be categorized). The likelihood that the neuropathology predicts the DLB clinical syndrome was low in 6\%, intermediate in $13 \%$ and high in $13 \%$ of all 304 subjects. In the latter two groups, $77 \%$ were demented, $35 \%$ had at least one extrapyramidal symptom, and $15 \%$ had visual hallucinations. Surprisingly, DLB clinical features associated better with high neurofibrillary stage than with diffuse neocortical LRP. Moreover, the neurofibrillary stage, substantia nigra (SN) neuron loss and grade of Lewy neurites (LNs) in hippocampal CA2-3 region, each showed a significant association with the extent of LRP. In conclusion, the neuropathological DLB in this very elderly population was common, but the clinical symptoms tended to associate better with severe neurofibrillary pathology than with extensive LRP.

Key words: Dementia with Lewy bodies, Lewy-related pathology, $\alpha$-synuclein, Alzheimer's disease, Neurofibrillary pathology, Population-based study, Aging. 


\section{INTRODUCTION:}

Dementia with Lewy bodies (DLB), the most frequent $\alpha$-synucleinopathy and the second most common cause of primary neurodegenerative dementia in elderly, is neuropathologically characterized by the presence of Lewy-related pathology (LRP) i.e. Lewy bodies (LBs) and dystrophic Lewy neurites (LNs) in the brainstem and cerebral cortex. The consortium on DLB (CDLB) international workshop proposed consensus guidelines for diagnosis of DLB in 1996 [1], which were revised in the Third CDLB in 2005 [2]. In addition to advice for categorizing LRP (to brainstem-predominant, limbic and diffuse neocortical type) based on density and regional distribution of LBs and LNs detected with $\alpha$-synuclein $(\alpha \mathrm{S})$ immunohistochemistry, the Third CDLB guidelines proposed a probability statement of neuropathologic findings associating with the DLB clinical syndrome [2]. This statement takes into account the extent of LRP and the often co-occurring Alzheimer type pathology. The type of LRP can be assigned according to the Third CDLB guidelines to many cases, but at the same time considerable number of cases remain nonassignable cases $[3,4]$. In addition, the association between the pathology and clinical features has been variable based on the study designs. Results of case-control studies have seemed to support the proposed probability statement [2], while studies based on unselected cases have indicated weaker association between the LRP and clinical features of DLB [4-6]. These discrepant results have thus challenged the probability statement and the significance of LRP. Besides LRP and Alzheimer type pathology, the occurrence of clinical features in DLB may depend on other factors such as neuronal loss. The grade of neuronal loss in the SN has been demonstrated to be one of the distinguishing factors between Parkinson disease (PD) and pre-symptomatic PD [7, 8].

The aims of this study were to (a) evaluate the frequency of subjects with neuropathologic DLB, categorized according to the revised consensus guidelines by the Third CDLB [2], (b) to evaluate how valid the probability statement regarding the clinico-pathologic associations was and 
(c) to determine the relevance of neuronal loss in the SN to the LRP and clinical features in this very elderly population.

\section{MATERIALS AND METHODS:}

\section{The study population}

The Vantaa 85+ study cohort includes all 601 residents of Vantaa, a town in Southern Finland, who were at least 85 years of age on April 1, 1991. The study design has been described in detail earlier $[9,10]$. Results of consented autopsies with neuropathologic examinations and clinical data were available in 304 (54\%) of the 565 eligible study subjects, who died during the ten-year follow-up by April 1, 2001 (Table 1). This prospective, population-based cohort study has been approved by the ethics committee of the Health Centre of the city of Vantaa and by the National Authority for Medicolegal Affairs, Helsinki, Finland.

\section{Clinical assessment}

A detailed clinical assessment protocol has been described earlier [10]. Subjects were considered demented if they fulfilled the guidelines in the Diagnostic and Statistical Manual of Mental Disorders (third edition, revised; DSM III-R), and the duration of the dementia had been at least 3 months. In this study, the information regarding the presence of rigidity and hypokinesia was derived from the last clinical examination prior to death and regarding the occurrence of visual hallucinations if reported at any time during the follow up. The mean time from the last clinical examination to death was 16 months (SD 13 months).

\section{Neuropathologic assessment}

The brains of the autopsied subjects were fixed in phosphate buffered $4 \%$ formaldehyde for at least 2 weeks before sampling. Tissue samples were obtained following recommendations 
of the First CDLB international workshop [1] for assessing LRP, and for the neuropathologic staging of neurofibrillary Alzheimer's disease (AD) changes [11]. Thus, the brain samples included the SN, middle frontal gyrus, superior and middle temporal gyri, inferior parietal lobule, anterior cingulate gyrus, left and right hippocampus (at the level of the lateral geniculate body), transentorhinal and entorhinal cortex (at the level of the mamillary bodies), and the primary and secondary visual cortices (the striate area, parastriate field and peristriate region all represented in the same specimen).

The Braak stage of neurofibrillary pathology was assessed as described earlier [9]. Sections of SN stained with the haematoxylin and eosin (H\&E) method and sections of SN and right hippocampus stained with antibodies against $\alpha \mathrm{S}$ were used to screen for LRP. In one subject without a sample of $\mathrm{SN}$, dorsal motor nucleus of vagus and locus coeruleus were used instead. If any LRP was detected in screened areas, immunohistochemical staining for $\alpha \mathrm{S}$ was performed on cortical samples obtained from the temporal, frontal, and parietal lobes and cingulate gyrus, as recommended by the First CDLB [1]. For immunohistochemistry, 98\% formic acid (5 minutes) and microwaving were used for pretreatment, and sections were incubated overnight with the primary $\alpha \mathrm{S}$ antibody (Transduction Laboratories, Lexington, KY, USA, clone 42, mouse monoclonal, diluted 1:800). Otherwise immunohistochemistry was done as described earlier in detail [12].

The load of LRP was semiquantitatively scored (none $=0$, mild $=1$, moderate $=2$, severe $=3$ and very severe $=4$ ), and followed by the assignment of the type of LRP (none, brainstempredominant, limbic, diffuse neocortical) for every subject according to the Third CDLB guidelines for diagnosis of DLB [2]. Since 45\% (50/110) of the subjects with LRP did not precisely fit to any existing category of LRP, we modified the criteria by allowing score 1 for parietal lobe within the limbic type of LRP (17 subjects), and score 4 to any area within the diffuse neocortical type of LRP (8 subjects). Further, 3 subjects with otherwise brainstempredominant but with score 1 in one neocortical area were kept in the brainstem-predominant type 
of LRP, and 9 subjects with otherwise limbic but with score 0 in transentorhinal cortex were included in the limbic type of LRP. Regardless of the modification, 13 subjects with score 1 LRP confined to the hippocampal-transentorhinal region (without LRP in SN, cingulate gyrus or neocortex) were not assignable to any of the existing LRP category according to the Third CDLB guidelines. Thus, these 13 were regarded as a category of their own, which was excluded from the association analyses. Based on the type of LRP and the Braak stage for neurofibrillary pathology, the likelihood that observed neuropathology would be associated with the clinical syndrome of DLB was determined as recommended into low, intermediate and high [2].

Additional investigations included a semiquantitative grading of the cell loss/atrophy in the ventrolateral tier of SN pars compacta. The grade, extending from none (0) to severe (3), was determined preferentially by assessing the loss of pigmented neurons, but in borderline cases also the amount of extraneuronal pigment was considered (Figure 1). The other additional investigation was the semiquantitative assessment of the LNs in the hippocampal CA 2-3 region as previously recommended [13].

\section{Statistical methods}

Statistical analyses were conducted with SPSS 15.0 program for Windows (SPSS Inc., Chicago, IL). For categorical variables the differences between the groups were analyzed with Chi-Squared or Fisher's exact tests or with Chi-Squared or exact tests for linear trend. Logistic regression analysis was used to assess association of several predictor variables with dichotomous dependent variables. $p$-value $<0.05$ was considered significant.

\section{RESULTS}

\section{Demographic characteristics (Table 1)}

Of the neuropathologically examined subpopulation ( $n=304), 64 \%$ were demented and $83 \%$ were women. The mean age at death was 92.4 years $(92.5$ years for demented, and 92.1 years 
for non-demented). There were somewhat more demented subjects in the neuropathologically examined subpopulation compared to the whole study population (64\% vs. 58\%), but regarding the other parameters the subpopulation and the whole study population were essentially identical (Table 1).

\section{Lewy-related pathology}

LRP was present in the SN and/or hippocampal-transentorhinal region in 36\% (110/304) of the autopsied subjects (Table 2). Thirteen (4\%) subjects had LRP confined to the hippocampaltransentorhinal region. The remaining 97 LRP positive subjects (32\%) - later in the text LRP refers to these subjects only - were categorized as follows: 8 subjects (3\% of 304) were brainstempredominant, $42(14 \%)$ were limbic, and $47(15 \%)$ were diffuse neocortical type when the Third CDLB criteria with our modifications were applied (Table 2). The LRP was somewhat more common in men (38\%) than in women (31\%), but the difference was not significant. Most men with LRP had diffuse neocortical type (65\%), while in LRP positive women the frequencies of limbic (47\%) and diffuse neocortical (44\%) type were rather equal (Table 2). Neither the category nor the frequency of LRP was influenced by age in this very elderly population (Table 2).

\section{Braak stage of neurofibrillary pathology and LRP}

Among the 304 neuropathologically examined subjects, there were 90 subjects (30\%) with Braak neurofibrillary stage 0, I or II, 142 subjects (47\%) with stage III-IV and 72 subjects (24\%) with stage V-VI. When subjects with LRP and Braak stage 0-II were considered pure DLB subjects, this group of 26 subjects (27\% of LRP positive) included 2 with brainstem-predominant, 12 with limbic, and 12 with diffuse neocortical type of LRP (Table 3).

The Braak stage associated significantly with the extent of LRP $(\mathrm{p}=0.011$, the chi squared test for linear trend, Figure 2). Anatomically, there was a significant association between the regional LRP score in transentorhinal cortex and the increasing Braak stage when applying the 
stage in three groups ( 0 -II, III-IV, V-VI, $p=0.027)$, but there was no such association between the regional LRP score and Braak stage in any other brain area.

When taking both LRP and Alzheimer type neurofibrillary pathology into consideration, the likelihood that the observed neuropathology was presented with the DLB clinical syndrome was low in $19(6 \%)$, intermediate in $39(13 \%)$ and high in $39(13 \%)$ subjects (Table 2). Men had slightly lower Braak stages compared to women, and at the same time more extensive LRP giving significantly higher likelihoods for men when comparing men vs. women ( $\mathrm{p}=0.006$, Table 2$)$.

\section{Clinical relevance of LRP and neurofibrillary pathology}

Dementia. Both the LRP and Braak stage had an effect on the likelihood of dementia (Table 3). All subjects with diffuse neocortical type and Braak stage V-VI pathology were demented, but there was also an independent association between dementia and LRP ( $p=0.021)$ and between dementia and Braak stage $(\mathrm{p}<0.001)$ when adjusted for gender and age at death. Among 26 pure DLB subjects (i.e. with LRP and Braak stage $0-\mathrm{II}$ ), 50\% of both brainstempredominant and limbic, and $75 \%$ of the diffuse neocortical type subjects were demented. Subjects with diffuse neocortical disease were 4.3 times (OR, 95\% CI 1.7-11.2) more likely to be demented than subjects without LRP when in the logistic regression analysis adjusted for gender, age at death, and Braak stage (Table 4). Among the 194 subjects with neurofibrillary pathology only, $48 \%$ of those with Braak stage 0 -II, $53 \%$ with stage III-IV, and $88 \%$ with stage V-VI were demented (Table 3). The probability for being demented was 8.0 times (OR, 95\% CI 3.1-20.9) higher in subjects with Braak stage V-VI compared to subjects with Braak stage 0-II pathology when adjusted for gender, age at death and type of LRP (Table 4).

A significant association was also found between dementia and the density of dystrophic LNs in the hippocampal CA2-3 region ( $p=0.009$, adjusted for gender and age at death). Although the overall significance of LNs was lost if adjusted for Braak stage, the association of the highest score of LNs with dementia remained significant (OR 8.8; 95\% CI 1.1-71.1; $p=0.041$ ). 
Anatomically, the presence of dementia increased with the increase of regional LRP score in the transentorhinal cortex independently of the Braak stage ( $p=0.039$, adjusted also for gender and age at death). In the other brain areas there was no significant association between dementia and LRP score regardless of the Braak stage.

Extrapyramidal symptoms (EPS) and visual hallucinations. The proportion of subjects with extrapyramidal symptoms (rigidity and/or hypokinesia) tended to increase along with the extension of LRP from limbic to the diffuse neocortical type, but also along with the increasing Braak stage (Tables 3 and 5). Thus, of the subjects with pure diffuse neocortical type of DLB (Braak stage 0-II), 25\% manifested rigidity and hypokinesia, while the corresponding figures of the subjects with Braak stage V-VI and without LRP were $34 \%$ and 24\% (Table 5). The type of LRP associated significantly with rigidity ( $p=0.042$ ), as well with hypokinesia ( $p=0.016)$, but only the association with hypokinesia remained significant when adjusted, in addition to gender and age at death, for Braak stage $(p=0.027)$ and additionally for dementia $(p=0.022)$. However, the association with hypokinesia was based on the brainstem-predominant type of LRP which category included only 8 subjects (Table 4). At the same time, Braak stage did not associate with hypokinesia $(p=0.11)$ but associated with rigidity $(p=0.031)$, though the significance was lost if adjusted for dementia $(p=0.34)$ or for the type of $\operatorname{LRP}(p=0.13)$. It is worth noting, however, that when both pathologies taken into account, the subjects with diffuse neocortical type and subjects with Braak stage V-VI both had more or less borderline association with rigidity ( $p=0.063$ and $p=0.075$ ), but only subjects with Braak stage V-VI showed almost significant association with hypokinesia ( $p=0.054$, Table 4$)$. The LRP score in SN was not associated with rigidity $(p=0.17)$ nor with hypokinesia $(p=0.33)$.

Visual hallucinations reported in 53 subjects, associated with dementia regardless of the Braak stage or the type of LRP (OR 4.1; 95\% CI 1.7-10.0; $p=0.002$ ) and with Braak stage independently of the presence of dementia $(p=0.041)$, but no association was seen with the type of LRP ( $p=0.78$ ). The probability for being presented with visual hallucinations was more than three 
times higher in subjects with Braak stage III-IV or V-VI compared to subjects with Braak stage 0II pathology when gender, age at death, type of LRP and dementia all taken into consideration (Table 4).

Likelihood categories and clinical symptoms. When analyzing the likelihood categories assessed according to the Third CDLB guidelines [2] and the clinical symptoms, dementia was most common in the intermediate-likelihood category (85\% demented), and least common in the high-likelihood category (69\% demented, Table 3). Rigidity, hypokinesia and visual hallucinations were most common in the low-likelihood category, within which $47 \%$ manifested rigidity, 37\% hypokinesia, and 26\% visual hallucinations (Table 5). The subjects with intermediate and high likelihood of DLB are proposed to be regarded as neuropathologic DLB [3], within this group of our subjects, $77 \%$ were demented, $35 \%$ had at least one extrapyramidal symptom, and 15\% had visual hallucinations (Tables 3 and 5).

\section{Findings related to neuronal loss in the $\mathrm{SN}$}

Only $7(2 \%)$ of the 303 with SN available did not have any neuron loss in the pars compacta of SN, $161(53 \%)$ had mild, $115(38 \%)$ had moderate and $20(7 \%)$ had severe neuron loss. There was a significant positive association between the grade of SN neuron loss and the LRP score in SN $(p<0.001$, Figure 3$)$ and the type of LRP $(p<0.001)$. These associations remained significant even if subjects with the Braak stage V-VI were excluded, most likely because SN neuron loss did not associate with Braak stage $(p=0.37)$.

The grade of SN neuron loss associated with rigidity ( $p=0.036)$, most strongly in the severe neuron loss group compared to others (OR 3.27; 95\% CI 1.30-8.27; $p=0.012$, adjusted for gender and age at death). But somewhat unexpectedly no association was seen between the grade of SN neuron loss and hypokinesia $(p=0.57)$. 


\section{DISCUSSION}

\section{General findings}

In this study, we evaluated the burden of neuropathologic DLB and related clinical symptoms in a large prospective, population-based autopsy cohort of very elderly age group, applying the Third CDLB criteria [2]. The presence of LRP in the brains of these subjects at least 85 years of age was common: even $20 \%$ of non-demented showed neuropathologic DLB classifiable to brainstem-predominant, limbic or diffuse neocortical type, while the corresponding figure for those with dementia was $38 \%$ and in the whole neuropathologically examined subpopulation $32 \%$. Furthermore, it is of interest that a great majority (92\%) of the LRP positive subjects had a more extensive, i.e. limbic or diffuse neocortical type of distribution. However, in present study neuropathologic DLB did not really predict the clinical symptoms commonly related with DLB: one fifth of the subjects with limbic or diffuse neocortical type were not demented and almost two thirds of them did not have a single EPS. Based on the likelihood categories [2], 26\% of our subjects had extensive $\alpha$-synucleinopathy corresponding to the intermediate and high likelihood, but neither did these predict the DLB clinical syndrome: almost one fourth were not demented and two thirds did not have a single EPS.

\section{Comparisons with previous studies}

The frequency of LRP in all screened areas in our study was $36 \%$, which is almost identical to the previously reported prevalence (37\%) in an elderly population (MRC CFAS study) from UK [14]. Our results are also consistent with those of a recently published population-based Hisayama study in which $31 \%$ of demented elderly Japanese individuals had LRP; among these $23 \%$ had limbic or diffuse neocortical type, and altogether $16 \%$ showed an intermediate or high likelihood that neuropathology was related to the clinical DLB syndrome [3]. The lower frequencies reported in the Hisayama study [3] may well be explained by the younger mean age of 
the subjects in that study (mean age at death for demented subjects in Hisayama study was 86.2 years vs. 92.5 years in our study).

DLB is reported to affect men with slight excess [15]. Our results are consistent with that impression, but it is important that our subpopulation included only $17 \%$ men. Nevertheless, this study suggests that neuropathologic DLB is slightly more frequent in men also among individuals aged 85 years and older, and men most often exhibit diffuse neocortical type of LRP. On the other hand, the presence of LRP in men seemed to be less often associated with the clinical symptoms than in women. Earlier studies have concluded that the frequency of LRP increases and LRP extends wider with age [3,16], even between the eighth and tenth decades [17]. In our study, we could not find the evidence - within this limited age range of very elderly subjects - for age being a risk factor for LRP. It may be that the frequency of LRP increases along with aging only until a certain age.

\section{Neuropathologic evaluation of the proposed categorization of LRP}

The neuropathologic diagnosis of DLB was based on the Third CDLB recommendation [2]. According to this, the types of LRP were defined as none, brainstem predominant, limbic and diffuse neocortical. Due to the overlapping scores in present staging scheme [2], and because the LRP score could vary significantly from one anatomic region to another, the determination of a "correct" type was problematic in almost half of the subjects. Actually, according to the revised criteria, some individuals could have been included in any of the proposed three types, depending on the interpretation. Some samples were particularly problematic - for example, according to the proposed guidelines, score 1 for LRP in the parietal lobe is allowed only for the diffuse neocortical type [2], in which it is expected that other neocortical areas were also affected. However, our subpopulation contained several individuals who showed score 1 LRP in the parietal lobe without any LRP in the other neocortical samples. On the other hand, 8 subjects had score 4 in the neocortex based on the suggested scoring scheme for LRP, compared to the 
expected "maximum" score of 3 in the Third CDLB criteria [2]. In addition, according to our results, it seems possible that there are also subjects who show focal LRP in the cerebrum without such pathology in the brainstem. The current categorization of LRP types does not acknowledge that possibility.

Other researchers have also had difficulties in defining the type LRP according the current guidelines, and modified criteria have been proposed [3, 18]. Further, Leverenz and colleagues suggest in their modified protocol to investigate only the frontal sample of the neocortical areas [18], which our findings argue against because we did not find any association between dementia and LRP score in the samples of frontal cortex. In contrast, the burden of LRP in the transentorhinal cortex associated with the increasing Braak stage, and also strongly with dementia regardless of the Braak stage, indicating that this sample may be useful when clinico-pathological association regarding dementia are evaluated in DLB patients.

\section{Clinico-pathological association}

The likelihood categories [2] in unselected autopsy studies have shown moderate accuracy for dementia and EPS [4]. It appears that the clinical DLB syndrome has a positive association to the extent of LRP and a negative association to the severity of Alzheimer type pathology $[2,6]$, i.e. subjects with pure DLB present more often with DLB core symptoms compared to those with severe concomitant Alzheimer type pathology. Especially those with extensive neurofibrillary pathology have been reported to show fewer clinical features of DLB, like visual hallucinations $[19,20]$. Our findings did differ from those results since visual hallucinations did associate significantly with the Braak stage but not with LRP. Interestingly, hypokinesia seemed to be associated with brainstem-predominant type of LRP and, more or less significantly, with Braak stage V-VI but not at all with diffuse neocortical DLB.

In our study rigidity was significantly associated with the neuron loss in SN but not with the LRP burden in SN. However, the most severe neuron loss of SN was seen in subjects with 
diffuse neocortical type of LRP who also had severe LRP burden in SN. There was no association between rigidity and the type of LRP if also Braak stage taken into account. In our study there was a borderline association between EPS and Braak stage V-VI as well between EPS and diffuse neocortical DLB, though these were lost if adjusted for dementia. Therefore, the competing effects of DLB and AD may well explain, why the association between the EPS and LRP in our study and in another population-based study [3] did not convincingly correlate with the likelihood categories [2]. Our results suggest that it might be helpful to take into account the neuron loss of SN while assessing whether the clinically detected rigidity was related with the LRP or Alzheimer type pathology (Braak stage).

The process of LRP formation has been suggested to be triggered by AD pathology [21]. Jellinger and colleagues have found correlation between the Braak Parkinson stage and Braak neurofibrillary AD stage $[22,23]$. The detected association between the extent of LRP and the Braak stage of neurofibrillary pathology in our study is in line with those results. These findings suggest interaction between $\alpha \mathrm{S}$ and tau, as has been suggested also based on the genetic studies [24]. Thus, hypothetically the end-stage of DLB would be diffuse neocortical distribution of LRP with severe neurofibrillary pathology. In this case, exclusion of subjects with frequent Alzheimer type pathology would misguide us in association analyses. Yet, the Third CDLB criteria of likelihood categories [2] are emphasized to find "pure" DLB behind the clinical features. Thus, in our very elderly subjects, high likelihood category did not predict the DLB clinical syndrome.

\section{Strengths and weaknesses of this study}

To the best of our knowledge, this is one of the first population-based studies focusing on LRP in very elderly age groups. The study population included all individuals of 85 years of age and older who lived in one town at one time-point, and thus there was no selection bias in the original cohort. The clinical follow-up examinations were done by a neurologist who used the same protocol throughout the study. The use of clinical and social care notes improved the 
accuracy of the diagnosis of dementia [25]. Autopsy frequency was over 50\% during the ten-year follow-up - a figure, which is one of the highest among population-based autopsy studies [26]. As shown in Table 1, there were hardly any differences between the autopsied subpopulation and all deceased during the follow-up period. The 6 percent-units difference in the frequency of subjects with dementia between the autopsied subpopulation and the whole study population has only a minor effect on the estimated frequency of LRP in the whole study population. We calculated the dementia-adjusted frequency of LRP by weighting the frequency of classifiable LRP among the demented (38\%) and non-demented (20\%) subjects in the autopsied subpopulation with the proportion of the demented (0.58) and non-demented (0.42) subjects in the whole study population. The result was $30 \%$ which is only 2 percent-units less than the proportion found among the autopsied subpopulation.

The clinical syndrome of DLB was not commonly known when the clinical study started in April 1991. This explains why the clinical examination protocol did not include assessing of the REM sleep behaviour, neuroleptic sensitivity, functional neuroimaging, and why no attention was paid to recognition of fluctuation in cognition [2]. For evaluating the parkinsonism and psychiatric symptoms we did not use the UPDRS or in the 1994 published NPI evaluation system, but the evaluation was done systematically based on the clinical standard of that time. Errors in the assessment of clinical features, such as delusions considered visual hallucinations, or inaccurate assessment of hypokinesia and rigidity are, of course, possible when assessing subjects in this oldest old age group, most of who represent with dementia and are confined to bed. This may have effect on our poor association between the DLB likelihood categories and clinical symptoms.

Since, all subjects of the neuropathologically examined subpopulation had died by April 2001, the sampling protocol followed the recommendations of the First CDLB in which a sample of amygdala was not included [27]. Thus, our sampling protocol did not follow exactly the more recent guidelines [2]. As both guidelines indicate that SN is always affected in DLB [1, 2], we 
used SN to screen for LRP in our neuropathologically examined subjects. Due to our interest in the frequency of LRP in the hippocampal CA 2-3, the other sample used for screening was hippocampus with the posterior edge of the transentorhinal cortex. Because we did not use amygdala or dorsal motor nucleus of vagus for additional screening, nor did examine the cortical samples of every subject, we may have missed some LRP positive brain areas. Nevertheless, it is unlikely that we have missed any or at least a significant number of cases of limbic or neocortical DLB. For example in the Hisayama study [3], only two of the 71 subjects with LRP had limbic or cortical pathology without LRP in SN or transentorhinal cortex. Furthermore, the other of these two subjects had score 1 in the parietal lobe (see also above!) only and was included in the noLRP group. The other had score 3 in the amygdala without any LRP in the cortical samples. The amygdala may show marked LRP without that pathology in any other brain area, especially when associated with AD [28]. The clinical impact of LRP in amygdala is still largely unknown [29], but a category of their own has been proposed to these "amygdala only" subjects $[6,18]$. We found 13 not classifiable cases with LRP restricted to the hippocampal-transentorhinal region. Further studies are needed to investigate, whether these are associated and combinable with the "amygdala only" subjects.

\section{Conclusions}

The Third CDLB guidelines [2] propose a method to define the likelihood that observed neuropathology accounts for DLB clinical syndrome. However, in very elderly people even extensive LRP is not necessarily accompanied by recognizable clinical symptoms, and the "correct" clinical symptoms are not necessarily associated with LRP. Thus, although the clinical diagnosis of DLB may be reliable if the disorder occurs in relatively pure form, the diagnosis is likely to be challenging in the very elderly people, who may have multiple pathologies in their brains. Further studies are needed to investigate how to distinguish clinically the effects of LRP from those of other pathologies, what is the pathogenic relationship between LRP and Alzheimer 
type pathology, and whether the age of subjects affects the threshold of appearance of the various clinical features of DLB.

\section{ACKNOWLEDGEMENTS}

We thank Tuija Järvinen for expert technical assistance and Dr. Auli Verkkoniemi for clinical evaluations during the baseline study of Vantaa 85+. This study was supported by the Alzheimer Foundation of Finland, Maire Taponen Foundation, Finnish Cultural Foundation, and Uulo Arhio Foundation.

\section{REFERENCES}

[1] McKeith IG, Galasko D, Kosaka K, Perry EK, Dickson DW, Hansen LA, Salmon DP, Lowe J, Mirra SS, Byrne EJ, Lennox G, Quinn NP, Edwardson JA, Ince PG, Bergeron C, Burns A, Miller BL, Lovestone S, Collerton D, Jansen EN, Ballard C, de Vos RA, Wilcock GK, Jellinger KA and Perry RH (1996) Consensus guidelines for the clinical and pathologic diagnosis of dementia with Lewy bodies (DLB): report of the consortium on DLB international workshop. Neurology 47, 1113-1124.

[2] McKeith IGFM, Dickson DW, Lowe JDM, Emre M, O'Brien JTDM, Feldman HCM, Cummings J, Duda JE, Lippa C, Perry EKDS, Aarsland D, Arai H, Ballard CG, Boeve B, Burn DJFRCP, Costa D, Del Ser T, Dubois B, Galasko D, Gauthier SFRCPC, Goetz CG, GomezTortosa E, Halliday G, Hansen LA, Hardy J, Iwatsubo T, Kalaria RNFRCP, Kaufer D, Kenny RA, Korczyn A, Kosaka K, Lee VMY, Lees A, Litvan I, Londos E, Lopez OL, Minoshima S, Mizuno Y, Molina JA, MukaetovaLadinska EB, Pasquier F, Perry RHDS, Schulz JB, Trojanowski JQ, Yamada M and for the Consortium on DLB (2005) Diagnosis and management of dementia with Lewy bodies: Third report of the DLB consortium. Neurology 65, 1863-1872. 
[3] Fujimi K, Sasaki K, Noda K, Wakisaka Y, Tanizaki Y, Matsui Y, Sekita A, Iida M, Kiyohara Y, Kanba S and Iwaki T (2008) Clinicopathological outline of dementia with Lewy bodies applying the revised criteria: the Hisayama study. Brain Pathol 18, 317-325.

[4] Parkkinen L, Pirttila T and Alafuzoff I (2008) Applicability of current staging/categorization of alpha-synuclein pathology and their clinical relevance. Acta Neuropathol 115, 399-407.

[5] Braak H, Rub U, Jansen Steur EN, Del Tredici K and de Vos RA (2005) Cognitive status correlates with neuropathologic stage in Parkinson disease. Neurology 64, 1404-1410.

[6] Fujishiro H, Ferman TJ, Boeve BF, Smith GE, Graff-Radford NR, Uitti RJ, Wszolek ZK, Knopman DS, Petersen RC, Parisi JE and Dickson DW (2008) Validation of the neuropathologic criteria of the third consortium for dementia with Lewy bodies for prospectively diagnosed cases. J Neuropathol Exp Neurol 67, 649-656.

[7] Dickson DW, Fujishiro H, DelleDonne A, Menke J, Ahmed Z, Klos KJ, Josephs KA, Frigerio R, Burnett M, Parisi JE and Ahlskog JE (2008) Evidence that incidental Lewy body disease is pre-symptomatic Parkinson's disease. Acta Neuropathol 115, 437-444.

[8] DelleDonne A, Klos KJ, Fujishiro H, Ahmed Z, Parisi JE, Josephs KA, Frigerio R, Burnett M, Wszolek ZK, Uitti RJ, Ahlskog JE and Dickson DW (2008) Incidental lewy body disease and preclinical Parkinson disease. Arch Neurol 65, 1074-1080.

[9] Polvikoski T, Sulkava R, Myllykangas L, Notkola IL, Niinisto L, Verkkoniemi A, Kainulainen K, Kontula K, Perez-Tur J, Hardy J and Haltia M (2001) Prevalence of Alzheimer's disease in very elderly people: a prospective neuropathological study. Neurology 56, 1690-1696.

[10] Barkhof F, Polvikoski TM, van Straaten EC, Kalaria RN, Sulkava R, Aronen HJ, Niinisto L, Rastas S, Oinas M, Scheltens P and Erkinjuntti T (2007) The significance of medial temporal lobe atrophy: a postmortem MRI study in the very old. Neurology 69, 1521-1527. 
[11] Braak H, Braak E (1991) Neuropathological stageing of Alzheimer-related changes. Acta Neuropathol 82, 239-259.

[12] Oinas M, Sulkava R, Polvikoski T, Kalimo H and Paetau A (2007) Reappraisal of a consecutive autopsy series of patients with primary degenerative dementia: Lewy-related pathology. APMIS 115, 820-827.

[13] Dickson DW, Ruan D, Crystal H, Mark MH, Davies P, Kress Y and Yen SH (1991) Hippocampal degeneration differentiates diffuse Lewy body disease (DLBD) from Alzheimer's disease: light and electron microscopic immunocytochemistry of CA2-3 neurites specific to DLBD. Neurology 41, 1402-1409.

[14] Zaccai J. Brayne C. McKeith I. Matthews F. Ince PG. MRC Cognitive Function,Ageing Neuropathology Study (2008) Patterns and stages of alpha-synucleinopathy: Relevance in a population-based cohort. Neurology 70, 1042-1048.

[15] McKeith IG, Fairbairn AF, Perry RH and Thompson P (1994) The clinical diagnosis and misdiagnosis of senile dementia of Lewy body type (SDLT). Br J Psychiatry 165, 324-332.

[16] Gibb WR, Lees AJ (1988) The relevance of the Lewy body to the pathogenesis of idiopathic Parkinson's disease. J Neurol Neurosurg Psychiatry 51, 745-752.

[17] Wakisaka Y, Furuta A, Tanizaki Y, Kiyohara Y, Iida M and Iwaki T (2003 Oct) Ageassociated prevalence and risk factors of Lewy body pathology in a general population: the Hisayama study.[see comment]. Acta Neuropathol 106, 374-382.

[18] Leverenz JB, Hamilton R, Tsuang DW, Schantz A, Vavrek D, Larson EB, Kukull WA, Lopez O, Galasko D, Masliah E, Kaye J, Woltjer R, Clark C, Trojanowski JQ and Montine TJ (2008) Empiric refinement of the pathologic assessment of Lewy-related pathology in the dementia patient. Brain Pathol 18, 220-224. 
[19] Merdes AR, Hansen LA, Jeste DV, Galasko D, Hofstetter CR, Ho GJ, Thal LJ and CoreyBloom J (2003 May 27) Influence of Alzheimer pathology on clinical diagnostic accuracy in dementia with Lewy bodies. Neurology 60, 1586-1590.

[20] Ballard CG, Jacoby R, Del Ser T, Khan MN, Munoz DG, Holmes C, Nagy Z, Perry EK, Joachim C, Jaros E, O'Brien JT, Perry RH and McKeith IG (2004 May) Neuropathological substrates of psychiatric symptoms in prospectively studied patients with autopsy-confirmed dementia with lewy bodies. Am J Psychiatry 161, 843-849.

[21] Saito Y, Ruberu NN, Sawabe M, Arai T, Kazama H, Hosoi T, Yamanouchi H and Murayama S (2004) Lewy body-related alpha-synucleinopathy in aging. J Neuropathol Exp Neurol 63, 742749.

[22] Wenning GK, Jellinger KA (2005) The role of alpha-synuclein and tau in neurodegenerative movement disorders. Curr Opin Neurol 18, 357-362.

[23] Jellinger KA, Attems J (2008) Prevalence and impact of vascular and Alzheimer pathologies in Lewy body disease. Acta Neuropathol 115, 427-436.

[24] Peuralinna T, Oinas M, Polvikoski T, Paetau A, Sulkava R, Niinisto L, et al. (2008) Neurofibrillary tau pathology modulated by genetic variation of alpha-synuclein. Ann Neurol 64, $348-352$.

[25] Polvikoski T, Sulkava R, Rastas S, Sutela A, Niinisto L, Notkola IL, Verkkoniemi A, Viramo P, Juva K and Haltia M (2006) Incidence of dementia in very elderly individuals: a clinical, neuropathological and molecular genetic study. Neuroepidemiology 26, 76-82.

[26] Zaccai J, Ince P and Brayne C (2006) Population-based neuropathological studies of dementia: design, methods and areas of investigation--a systematic review. BMC Neurol 6, 2. 
[27] McKeith IG, Perry EK and Perry RH (1999) Report of the second dementia with Lewy body international workshop: diagnosis and treatment. Consortium on Dementia with Lewy Bodies.

Neurology 53, 902-905.

[28] Hamilton RL (2000 Jul) Lewy bodies in Alzheimer's disease: a neuropathological review of 145 cases using alpha-synuclein immunohistochemistry. Brain Pathol 10, 378-384.

[29] Lopez OL, Becker JT, Sweet RA, Martin-Sanchez FJ and Hamilton RL (2006) Lewy bodies in the amygdala increase risk for major depression in subjects with Alzheimer disease. Neurology 67, 660-665.

\section{TABLES}


Table 1 Characteristics of the whole study population and the subpopulation of neuropathologically examined subjects

Vantaa 85+ Neuropathologic

Study population*

565

304

$\operatorname{Sex}(\mathrm{n}, \%)$

Men

$118(21 \%)$

$52(17 \%)$

Women

$447(79 \%)$

$252(83 \%)$

Dementia status (n, \%)

Demented

$326(58 \%)$

$239(42 \%)$

$196(64 \%)$

$108(36 \%)$

Frequency of dementia (n, \%)

Men

$63(53 \%)$

$30(58 \%)$

$263(59 \%)$

$166(66 \%)$

Age at onset (mean \pm SD)

$86.8( \pm 4.5)$

$87.1( \pm 4.5)$

Duration of dementia (mean \pm SD)

$5.2( \pm 3.5)$

$5.4( \pm 3.7)$

Age at death $($ mean $\pm \mathrm{SD})$

$91.9( \pm 3.6)$

$92.4( \pm 3.7)$

Age at death (n, \%)

85-89

90-94

$188(33 \%)$

$267(47 \%)$

$82(27 \%)$

$146(48 \%)$

$110(19 \%)$

$76(25 \%)$

Hospitalization at death

Hospital

$431(76 \%)^{* *}$

$238(78 \%)$

Nursing home

$99(18 \%)$

$55(18 \%)$

Home

$26(5 \%)$

$11(4 \%)$

\footnotetext{
* The study population includes all subjects who deceased during the ten-year follow-up time and had approved to participate in the study

${ }^{* *}$ For nine the hospitalization at the death not known
} 
Table 2 Distribution of Lewy-related pathology (LRP) and likelihood categories according to the age at death and gender.

Type of LRP

Age at death

All

Total $n=304$

Men $n=52$

Women $n=252$

85-89 years

Total $\mathbf{n}=82$

Men $n=15$

Women $\mathrm{n}=67$

90-94 years

Total $n=146$

Men $n=23$

Women $n=123$

$\geq 95$ years

Total $n=76$

Men $n=14$

Women $n=62$

\section{$50(61 \%)$}

$10(67 \%)$

$40(60 \%)$

\section{$95(65 \%)$

$$
13(57 \%)
$$

$13(57 \%)$
$82(67 \%)$

$42(68 \%)$

$\begin{array}{lll}\text {-None } & \begin{array}{l} \pm \text { Non- } \\ \text { classifiable }\end{array} & \begin{array}{l}{ }^{+} \text {Brain } \\ \text { predon }\end{array} \\ & & \\ & & \mathbf{8}(\mathbf{3 \%}) \\ \mathbf{1 9 4}(\mathbf{6 4 \%}) & \mathbf{1 3}(\mathbf{4 \%}) & 1(2 \%) \\ 30(58 \%) & 2(4 \%) & 7(3 \%) \\ 164(65 \%) & 11(4 \%) & \end{array}$

\section{$3(4 \%)$}

$$
0(0 \%)
$$$$
3(5 \%)
$$

$1(\mathbf{1 \%})$
$0(0 \%)$
$1(1 \%)$

$\begin{array}{ll}\mathbf{1 2}(\mathbf{1 5 \%}) & \mathbf{1 6}(\mathbf{2 0 \%}) \\ 2(13 \%) & 3(20 \%) \\ 10(15 \%) & 13(19 \%)\end{array}$

$\begin{array}{ll}\mathbf{4 2}(\mathbf{1 4 \%}) & \mathbf{4 7}(\mathbf{1 5 \%}) \\ 6(12 \%) & 13(25 \%) \\ 36(14 \%) & 34(13 \%)\end{array}$$$
1(1 \%)
$$

\section{$7(5 \%)$}

\section{$4(3 \%)$}

$1(4 \%)$
$6(5 \%)$

$1(4 \%)$

$3(2 \%)$

$\begin{array}{ll}\mathbf{2 2}(\mathbf{1 5 \%}) & \mathbf{1 8}(\mathbf{1 2 \%}) \\ 3(13 \%) & 5(22 \%) \\ 19(15 \%) & 13(11 \%)\end{array}$

$49(65 \%) \quad 3(4 \%)$

$7(50 \%) \quad 1(7 \%)$

$2(3 \%)$

Likelihood category

$\begin{array}{lll}\text { Low } & \text { Intermediate } & \text { High } \\ \text { likelihood } & \text { likelihood } & \text { likelihood }\end{array}$

$\begin{array}{lll}19(6 \%) & 39(\mathbf{1 3 \%}) & \mathbf{3 9}(\mathbf{1 3 \%}) \\ 1(2 \%) & 5(10 \%) & 14(27 \%) * \\ 18(7 \%) & 34(13 \%) & 25(10 \%) *\end{array}$

$1(2 \%)$

$34(13 \%)$

$25(10 \%) *$

$\begin{array}{lcc}\mathbf{4}(\mathbf{5 \%}) & \mathbf{1 4}(\mathbf{1 7 \%}) & \mathbf{1 1}(\mathbf{1 3 \%}) \\ 0(0 \%) & 1(7 \%) & 4(27 \%) \\ 4(6 \%) & 13(19 \%) & 7(10 \%)\end{array}$

$0(0 \%)$

$13(19 \%)$

$(10 \%)$

$\begin{array}{llr}\mathbf{1 0}(\mathbf{7 \%}) & \mathbf{1 7}(\mathbf{1 2 \%}) & \mathbf{1 7}(\mathbf{1 2 \%}) \\ 1(4 \%) & 4(17 \%) & 4(17 \%) \\ 9(7 \%) & 13(11 \%) & 13(11 \%)\end{array}$

-LRP negative subjects

\pm LRP confined to the hippocampal-transentorhinal region only

${ }^{+}$Regarded as LRP subjects in the text and analyses

*A significant difference in the presence of likelihood categories between men and women $(p=0.006)$ 
Table 3 Likelihood categories according to the type of Lewy-related pathology (LRP) and Alzheimer type neurofibrillary pathology (Braak stage), with frequency of dementia and at least one extrapyramidal symptom (EPS) i.e. rigidity and/or hypokinesia.

Alzheimer type neurofibrillary pathology

\begin{tabular}{|c|c|c|c|}
\hline Type of LRP & Braak stage 0-II & Braak stage III-IV & Braak stage V-VI \\
\hline $\begin{array}{l}\text { None } \\
n=194\end{array}$ & $n=60$ & $\mathrm{n}=101$ & $\mathrm{n}=33$ \\
\hline 112 demented $(58 \%)$ & 29 demented (48\%) & 54 demented $(53 \%)$ & 29 demented ( $88 \%)$ \\
\hline 54 with EPS (28\%) & 12 with EPS (20\%) & 27 with EPS (27\%) & 15 with EPS (45\%) \\
\hline $\begin{array}{l}\text { Brainstem-predominant } \\
\mathrm{n}=8\end{array}$ & $\begin{array}{l}\text { Low likelihood } \\
\mathrm{n}=2\end{array}$ & $\begin{array}{l}\text { Low likelihood } \\
n=3\end{array}$ & $\begin{array}{l}\text { Low likelihood } \\
n=3\end{array}$ \\
\hline 5 demented $(63 \%)$ & 1 demented $(50 \%)$ & 2 demented $(67 \%)$ & 2 demented $(67 \%)$ \\
\hline 5 with EPS (63\%) & 1 with EPS $(50 \%)$ & 2 with EPS (67\%) & 2 with EPS (67\%) \\
\hline $\begin{array}{l}\text { Limbic } \\
\mathrm{n}=42\end{array}$ & $\begin{array}{l}\text { High likelihood } \\
\mathrm{n}=12\end{array}$ & $\begin{array}{l}\text { Intermediate likelihood } \\
n=19\end{array}$ & $\begin{array}{l}\text { Low likelihood } \\
\mathrm{n}=11\end{array}$ \\
\hline 29 demented (69\%) & 6 demented $(50 \%)$ & 13 demented $(68 \%)$ & 10 demented $(91 \%)$ \\
\hline 12 with EPS (29\%) & 2 with EPS (17\%) & 5 with EPS $(26 \%)$ & 5 with EPS (45\%) \\
\hline $\begin{array}{l}\text { Diffuse neocortical } \\
n=47\end{array}$ & $\begin{array}{l}\text { High likelihood } \\
\mathrm{n}=12\end{array}$ & $\begin{array}{l}\text { High likelihood } \\
n=15\end{array}$ & $\begin{array}{l}\text { Intermediate likelihood } \\
\mathrm{n}=20\end{array}$ \\
\hline 41 demented $(87 \%)$ & 9 demented $(75 \%)$ & 12 demented $(80 \%)$ & 20 demented $(100 \%)$ \\
\hline 20 with EPS (43\%) & 5 with EPS (42\%) & 7 with EPS (47\%) & 8 with EPS (40\%) \\
\hline
\end{tabular}


Table 4 Associations of Lewy-related pathology and Alzheimer type neurofibrillary pathology with clinical features based on logistic regression analysis.

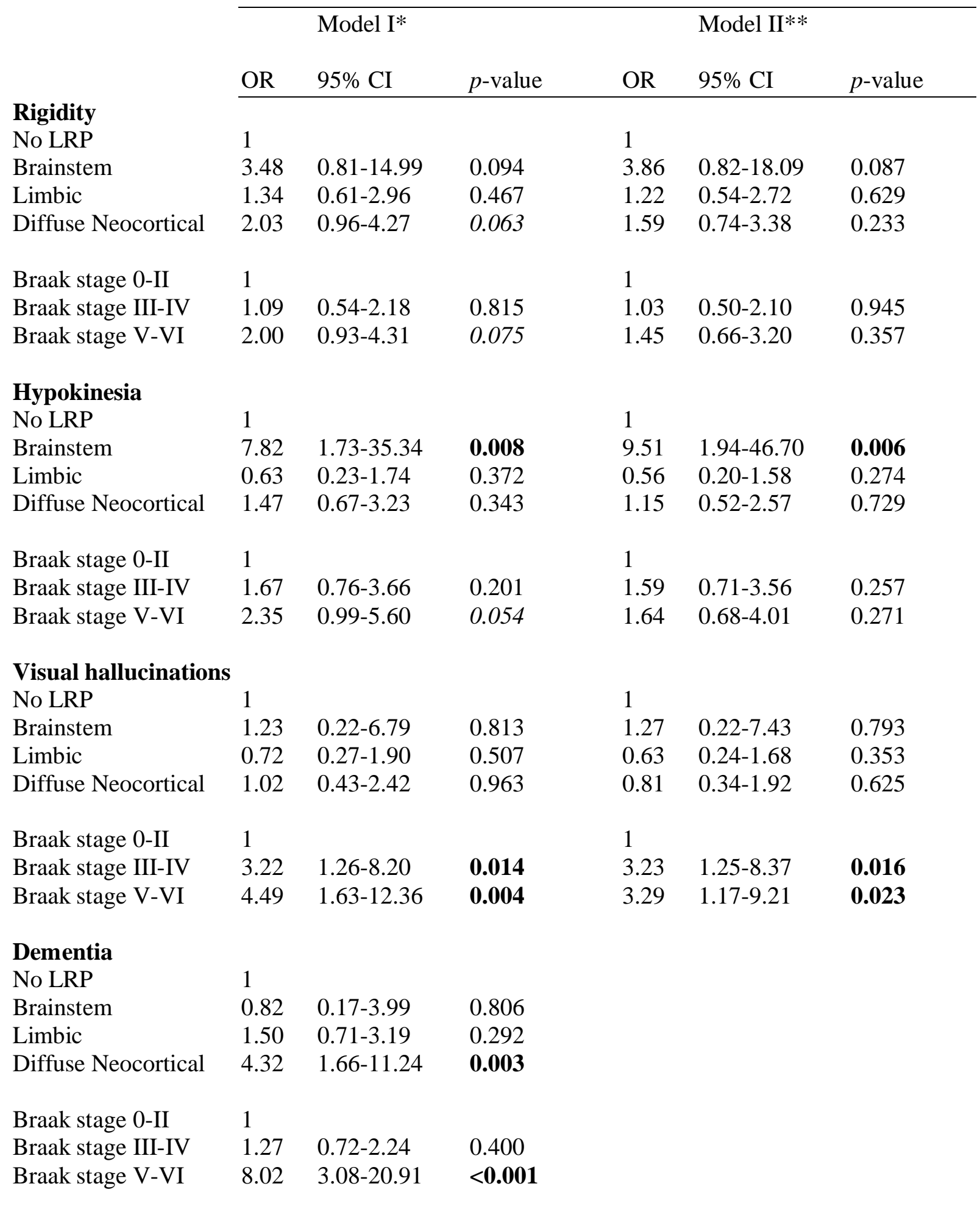

OR; Odds Ratio, 95\% CI; 95\% Confidence Interval

* The ORs are adjusted for gender and age at death, and mutually for type of LRP and Braak stage.

** The ORs are adjusted for gender, age at death, and dementia, and mutually for type of LRP and Braak stage. 
Table 5 The presence of the clinical features according to the type of LRP and Alzheimer type neurofibrillary pathology (Braak stage).

Type of LRP

None, $n=194$

Rigidity

Hypokinesia

Visual hallucinations

Brainstem-predominant, $n=8$

Rigidity

Hypokinesia

Visual hallucinations

Limbic, $\mathbf{n}=42$

Rigidity

Hypokinesia

Visual hallucinations

Diffuse Neocortical, $n=47$

Rigidity

Hypokinesia

Visual hallucinations
Braak stage 0-II

$\mathbf{6 0}(\mathbf{3 1 \%})$
$11(18 \%)$
$6(10 \%)$
$5(8 \%)$

$\mathbf{2 ( 2 5 \% ) +}$
$1(50 \%)$
$1(50 \%)$
$0(0 \%)$

$12(29 \%)^{*}$

$1(8 \%)$

$1(8 \%)$

$0(0 \%)$

\section{$12(26 \%) *$}

$3(25 \%)$

$3(25 \%)$

$1(8 \%)$
Braak stage III-IV

$\begin{array}{rr}\mathbf{1 0 1}(\mathbf{5 2 \%}) & \mathbf{3 3}(\mathbf{1 7 \%}) \\ 16(16 \%) & 11(33 \%) \\ 18(18 \%) & 9(27 \%) \\ 19(19 \%) & 10(30 \%)\end{array}$

$3(38 \%)+$

$2(67 \%)$

$2(67 \%)$

$1(33 \%)$

$3(38 \%)+$

$1(33 \%)$

$2(67 \%)$

$1(33 \%)$

\section{$19(45 \%) * *$}

$5(26 \%)$

$2(11 \%)$

$3(16 \%)$

\section{$11(26 \%)+$}

$5(45 \%)$

$2(18 \%)$

$3(27 \%)$

$15(32 \%) *$

$6(40 \%)$

$4(27 \%)$

$5(33 \%)$
$20(43 \%) * *$

$7(35 \%)$

$5(25 \%)$

$3(15 \%)$

+ Low likelihood category

** Intermediate likelihood category

* High Likelihood category 


\section{FIGURE LEGENDS}

Figure 1 Assessment of the neuron loss in the substantia nigra (SN). Hematoxylin and eosin (H\&E) staining (x200) illustrating the severing loss of pigmented neurons in SN.

Figure 2 Alzheimer type neurofibrillary pathology (Braak stage) according to the distribution of classifiable Lewy-related pathology ( $p=0.011$, the chi squared test for linear trend).

Figure 3 The neuron loss of the substantia nigra (SN) according to (A) Lewy-related pathology (LRP) score in SN ( $p<0.001$, the SPSS linear-by-linear exact test), and (B) the distribution of LRP $(p<0.001$, linear-by-linear exact test).

\section{FIGURES}


Figure 1

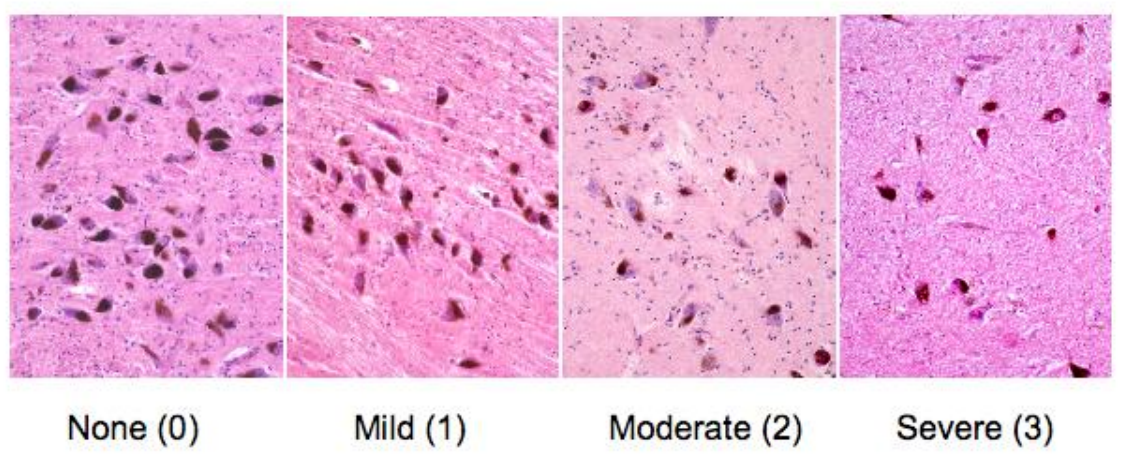

Figure 2

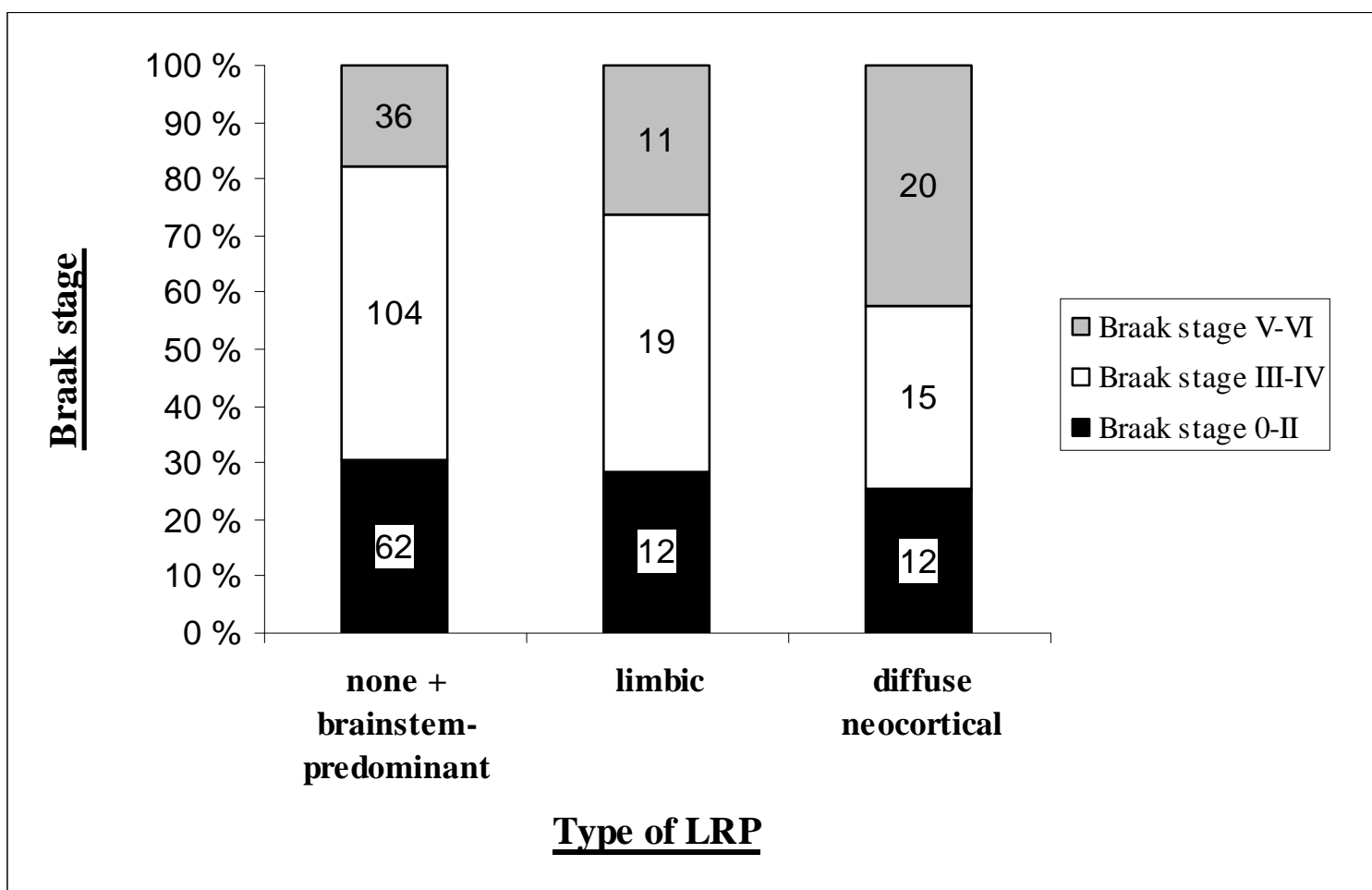


Figure 3
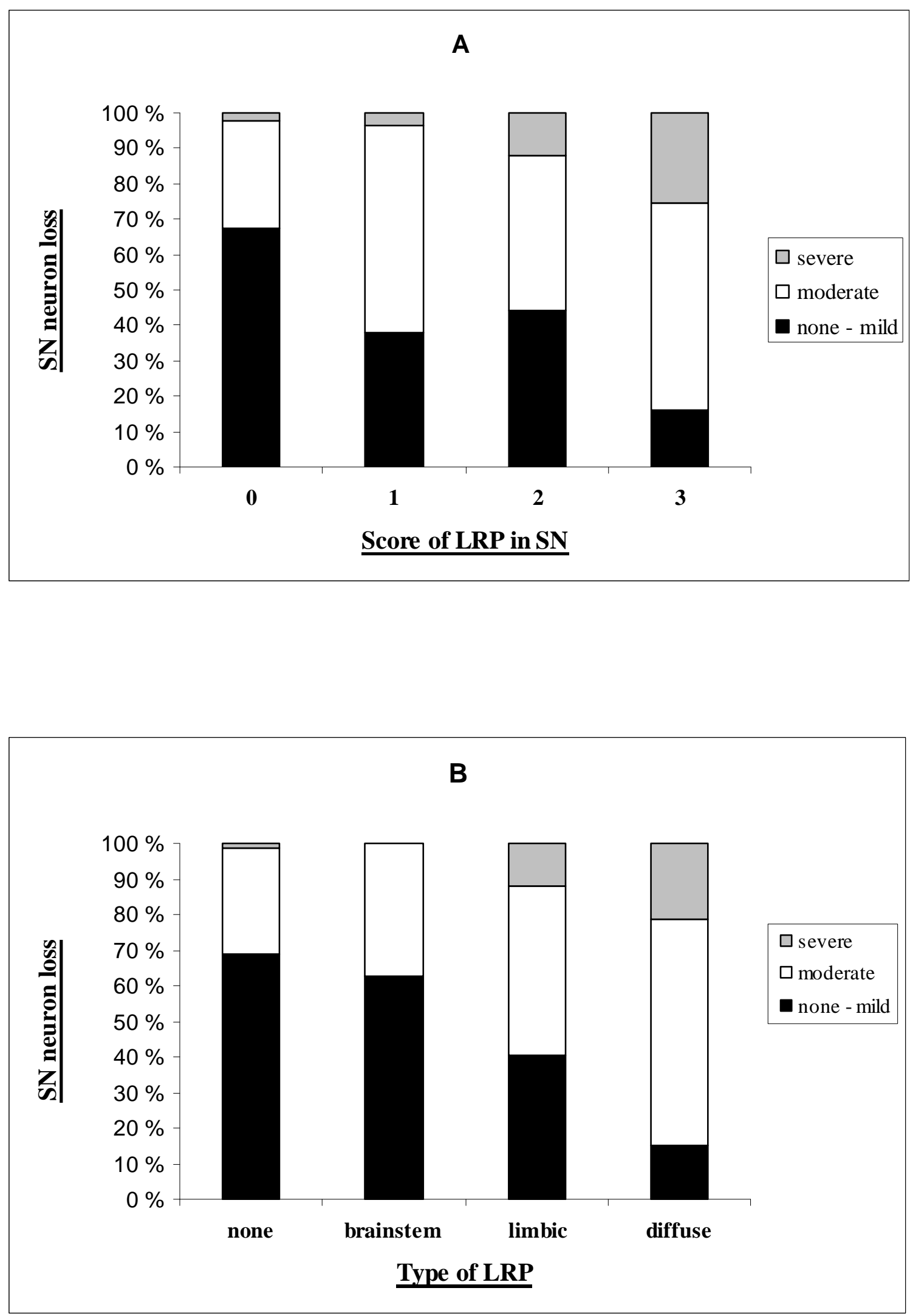